\title{
Induced prismatic effects due to poorly fitting spectacle frames
}

\author{
VR Moodley*, F Kadwa, B Nxumalo, S Penceliah, B Ramkalam and A Zama \\ *Discipline of Optometry, University of Kwa Zulu-Natal, Westville Campus, Private Bag X54001 Durban, 4000 \\ South Africa
}

<moodleyvr@ukzn.ac.za>

Received 20 June 2011; revised version accepted 2 November 2011

\begin{abstract}
To provide patients with the best vision possible practitioners undertake comprehensive visual examinations and optical dispensing procedures. Ensuring proper frame alignment is an important part of the spectacle dispensing process. Ideally, for maximum optical benefit, patients should look through the optical centers of their lenses. Rays entering a lens at any point other than the optical center will be deviated as long as the lens has power, thus inducing prismatic effects. These prismatic effects may lead to visual complaints such as asthenopia, blurry vision and headaches. The aim of the study was to investigate the prevalence of induced prismatic effects due to poorly fitting spectacle frames. A sample of 100 spectacle wearing subjects was selected as participants. Questionnaires were completed and any symptoms experienced recorded. The habitual spectacle position in the primary position of gaze was noted, pupil and optical centers marked and where relevant, induced prism was calculated. Data was analysed using the ANOVA Kruskal-Wallis and Mann-Whitney tests.

On initial observation of frame position, about $45 \%$ of subjects were wearing their spectacles in-
\end{abstract}

correctly. A comparison of the marked pupil centers in primary gaze and optical centers revealed that $100 \%$ of participants were found to not be looking through the optical centers of their lenses. Fifty one percent were within horizontal and $3.12 \%$ within vertical ANSI tolerances. Symptoms were reported by $50 \%$ of subjects experiencing base-out, $0 \%$ base-in and $47 \%$ vertical induced prismatic effects. However, no correlation existed between those subjects experiencing induced prism and symptoms reported. Prism adaptation may account for some participants being asymptomatic. Sixty three percent of subjects were not aware of the importance of properly fitted spectacle frames. Proper optical dispensing with associated patient education is necessary to achieve optimal optical benefits of spectacles and careful attention should be given to this aspect by practitioners. It is recommended that patients return periodically to their optometrists to have the frame alignment assessed and the fit modified if necessary. (S Afr Optom 2011 70(4) 168-174)

Key Words: induced prism, optical center, prismatic effects, prism adaptation

\section{Introduction}

A spectacle frame is the portion of the spectacles that holds the lenses, containing the ophthalmic prescription, in their proper position before the eyes. The various parts of the frame contribute to the final resting position of the frame on the face ${ }^{1}$. The bridge structure and adjustable nose pads cause the frame to rest higher or lower on the face and the lenses to be positioned at varying distances from the eyes. Temples help keep the spectacles from slipping down the nose when the bridge does not help much to support 
the frame or if the prescribed spectacles tend to be somewhat heavy ${ }^{1-3}$. Spectacles are very important, from an aesthetic point of view, to the person wearing them and the habitual wearer often needs as much help with frame selection as the neophyte. Aside from a frame being cosmetically appealing, the final frame choice should also provide the best optics with the prescription lenses ${ }^{4}$. Various factors such as overall facial features, frame design and colour, frame thickness and the patient's prescription should be taken into consideration when a frame is being selected ${ }^{1,3-5}$. Good optical dispensing practice must include evaluating the spectacles on the patient for function, fit and comfort. This will include aligning the frame before it is adjusted on the face to simplify angling for facial contours to ensure a good fit ${ }^{6}$. The practitioner usually adjusts the spectacles for the correct position to be attained on the face. A proper frame design and fit would be one that allows the distance optical center of the lenses to be positioned directly in front of the pupil.

The anatomical interpupillary distance (IPD) is defined as the distance between the centers of the entrance pupils of the eyes ${ }^{1,7-9}$. According to Borish ${ }^{7}$, the usual method of determining the IPD involves the use of a millimeter ruler to measure the distance between the centers of the subject's pupils. In the event that the exact center of the pupil is difficult to estimate, the IPD is measured from the nasal edge of one pupil to the temporal edge of the other provided that the two pupils are equal in size ${ }^{1,7-9}$. Potential errors when taking IPD include errors of parallelism due to the large difference between the observer and the patient's IPD, inappropriate distance between observer and patient and improper placement of the ruler before the patient's face ${ }^{8}$. The main reason for measuring the IPD is to align the optical centers of ophthalmic lenses on the visual axis of each eye and failure to do so results in unwanted induced prismatic effect which may lead to eyestrain and asthenopia ${ }^{9-11}$.

Pantoscopic tilt or angle is defined as the angle between the optical axis of the lens and the visual axis of the eye in the primary position or as the angle between the vertical plane of the face and the positioning of the glasses ${ }^{7-10}$. Most spectacles are fitted with downward tilts of about 7-10 degrees to the visual axis, meaning the top of the spectacle lens is approximately 10 degrees forward when compared to the bot- tom. Since the top of the spectacles are intentionally tilted forward, this helps to achieve a place that allows a more constant distance between the back surface of the spectacle lens and the front surface of the cornea $^{10}$. A dispensing rule of thumb used is to drop the optical centre by $0.5 \mathrm{~mm}$ for every 1 degree of pantoscopic tilt ${ }^{11}$. However, anecdotal evidence suggests that in practice, this position of the optical centre is often obtained with no specific instruction on the prescription order, as modern cosmetic dispensing dictates that the frame occupies a position such that the centre of the pupil lies 4-8 $\mathrm{mm}$ above the horizontal centre line.

Face-form tilt or wrap is the inclination of the temporal edge of the lens towards the face, ensuring that the frame front follows the natural frontal curvature of the skull12. This curve serves both the cosmetic purpose of improving the frame appearance and the optical purpose of aligning both lens surfaces with the wearer's line of sight ${ }^{1}$. When prescription lenses are ordered, the interpupillary distance is routinely measured. In addition, the eye size (measured from outermost edges of lenses horizontally) and bridge size (the distance between lenses) may also be measured and added together, a measurement termed the frame $\mathrm{PD}^{11,13}$.

After the optical laboratory generates the prescription in a spectacle lens blank, the finished lens must be cut in such a way that 1) it fits properly into the spectacle frame and 2) the optical center of the lens coincides with the visual axis that passes through the pupil center, a process termed centration ${ }^{10-11,13}$. It is very important that the optical centre of each lens should occupy the desired position relative to the pupil of the wearer's eye ${ }^{13}$. Accurate pupillary (facial PD) and centration distance (frame PD) measurements are required for this process. If the centration is not properly accomplished, the two centers will not coincide in one or both lenses, resulting in unnecessary prism (induced prism) ${ }^{11}$.

Induced prism is defined as the prismatic effect created when the patient's visual axis does not pass through the optical centre of an ophthalmic lens ${ }^{12}$. A light ray will pass through a lens without deviation only if it enters along the optic axis. Those parallel incident rays entering a lens at points other than the optical center will be deviated as long as the lens has refracting power, creating prismatic effects, so termed 
due to the lens having the characteristic effect of a prism $^{13}$. Patients will experience this effect when looking through part of a spectacle lens other than its optical center. The amount of induced prism depends upon the power of the spectacle lens and the decentration or optical center displacement from the pupil center ${ }^{11-14}$. Prism is frequently intentionally prescribed by the clinician in spectacle lenses to eliminate diplopia or to ease asthenopia. This prism may be incorporated by the application of Prentice's Rule $(\mathrm{P}=\mathrm{cF})$, which translates to each centimeter of decentration of a lens resulting in one prism diopter of deviation of light for each diopter of lens power at a distance of one metre ${ }^{14-15}$. In the equation $\mathrm{P}=\mathrm{cF}$, $\mathrm{P}$ is prism measured in prism diopters (pd), c is optical centre displacement measured in centimeters $(\mathrm{cm})$ and $\mathrm{F}$ is lens power measured in diopters (D). Jalie ${ }^{16}$ describes the application of Prentice's rule for horizontal and vertical meridians and the methodology to be applied when the cylinder axis is oblique and the resultant oblique prism has to be resolved into vertical and horizontal components.

The prism induced by decentration of ophthalmic lenses will also influence binocular vision and stereopsis which are important aspects of visual functioning ${ }^{16-17}$. Jiménez et al17 showed that decentration changes fusional convergence and can thereby alter certain aspects of binocular vision, such as fusion, resulting in eye fatigue or headaches. Fogt and Jones ${ }^{18}$ demonstrated that the prismatic effect of spectacle lenses results in inappropriate extra-retinal eye-position information in a dark environment and Fry and Kent ${ }^{19}$ found a deterioration of stereoacuity when they used base in and base out prisms to induce changes in convergence.

Du Toit et al ${ }^{20}$ investigated the amount of induced horizontal and vertical prism that could comfortably be tolerated with the use of ready-made spectacles. Nine participants were each given plano spectacles to wear with differing prism power for eight hours. If visual discomfort could not be tolerated, participants removed the spectacles, noting duration of wear and reason for discontinuation. Distance and near visual comfort were rated, and participants were asked if they would be able to adapt to wearing the spectacles. The highest prism powers that is (1 pd up, $2 \mathrm{pd}$ out, $2 \mathrm{pd}$ in) could not be worn for eight hours by the majority of the participants. Comfort ratings for near vision were statistically significantly different when the highest prism power was compared with each of the lower powers. The results of their study ${ }^{20}$ showed that vertically, the comfort ratings for the control and the $0.5 \mathrm{pd}$ were different from the $1 \mathrm{pd}$, whilst horizontally the comfort ratings for the control, $0.5 \mathrm{pd}$ and $1 \mathrm{pd}$ were all different from the $2 \mathrm{pd}$. The study concluded that most spectacle wearers would likely comfortably tolerate $\leq 0.5 \mathrm{pd}$ vertical, $\leq 1.0 \mathrm{pd}$ out, or $\leq 1.0 \mathrm{pd}$ in induced prism. A guide to the maximum interpupillary distance/optical center distance disparities likely to be comfortably tolerated with varying spectacle powers was formulated. Powers -1.50 to $+1.50 \mathrm{D}$ are unlikely to have sufficient lens decentration to cause discomfort ${ }^{20}$.

The allowed tolerances on prismatic power depends on whether the lens is glazed or unglazed, the lens is single vision, multifocal or progressive, prismatic effect at the centration point or prescribed prism is being measured and the prism is horizontal or vertical.

Table 1: Tolerances for glazed lenses incorporating less than $2 \mathrm{D}$ of prescribed prism ${ }^{21}$.

\begin{tabular}{|l|l|l|}
\hline Material lens powers (D) & \multicolumn{1}{|c|}{ Horizontal Tolerance } & \multicolumn{1}{|c|}{ Vertical Tolerance } \\
\hline Both lenses: power <2 D & $\begin{array}{l}0.25 \mathrm{pd} \text { and } 2.0 \mathrm{~mm} \\
\text { displacement }\end{array}$ & $\begin{array}{l}0.25 \mathrm{pd} \text { and } 1.0 \mathrm{~mm} \text { total } \\
\text { displacement }\end{array}$ \\
\hline Both lenses: power $\geq 2 \mathrm{D}$ & $\begin{array}{l}0.45 \mathrm{pd} \text { and } 2.0 \mathrm{~mm} \text { total } \\
\text { displacement }\end{array}$ & $\begin{array}{l}0.25 \mathrm{pd} \text { and } 1.0 \mathrm{~mm} \text { total } \\
\text { displacement }\end{array}$ \\
\hline One lens <2 D and one lens & $\begin{array}{l}0.12 \mathrm{pd} \text { and } 2.0 \mathrm{~mm} \\
\text { displacement }\end{array}$ & $\begin{array}{l}0.12 \mathrm{pd} \text { and } 1.0 \mathrm{~mm} \text { total } \\
\text { displacement }\end{array}$ \\
\hline
\end{tabular}


Tolerance set by the American National Standards Institute (ANSI): ${ }^{22}$

In 1979 the ANSI released a set of standards including vertical prism up to $1 / 3$ prism diopter or 1 mm decentration of the optical center was considered acceptable and horizontal prismatic imbalance of $2 / 3$ prism diopter or $2.5 \mathrm{~mm}$ decentration was considered acceptable.

Prism adaptation is considered a form of procedural learning 23 . When prisms are placed in front of the eyes, the entire visual field is displaced and the adaptation that occurs is a phenomenon in which the motor system adapts to new visuospatial coordinates imposed by the displaced visual field. The degree and strength of the adaptation can be measured by the spatial deviation of the motor actions in the direction opposite to the visual displacement imposed by the prisms, a phenomenon known as after-effect. Fernández-Ruiz and Díaz ${ }^{23}$ in their study on prism adaptation required subjects to throw balls to a target in front of them before, during, and after lateral displacement of the visual field with prismatic spectacles. The results showed that the adaptation process is dependent on the number of interactions between the visual and motor system, and not on the time spent wearing the prisms.

Osuobeni ${ }^{9}$ found that prismatic effects lead to various symptoms, such as asthenopia, blurry vision and diplopia. It is thus useful to take these prismatic effects into account when a patient wearing spectacles presents with such symptoms. In these instances, it may be incorrect to assume that the symptoms are only due to an incorrect refraction having been performed. Additionally, irrespective of how accurate the refraction has been, if the lenses (single vision, bifocals or varifocals) are improperly positioned before the eyes, the finished product will be inferior from a quality of vision perspective. It is therefore important that proper frame fitting and regular adjustments of spectacle frames by an optometrist or dispensing optician occurs with the aim of alleviating symptoms of eyestrain.

Spectacle frames also warp out of shape due to the body heat of the wearer, particularly at the bridge piece, causing the frames to bow slightly forwardly and the temple pieces to spread outwardly. Exposure to temperatures above 60 degrees Celsius, such as the intense heat of the car dashboard or sauna bath, leads to spectacle frame warpage as well as loosening of the lenses ${ }^{24}$. This can result in a loose fit of the frame and temple pieces, which permits the frames to slip down the nose, causing discomfort and distorted vision through the lens

Ophthalmic dispensing manuals and textbooks usually highlight the importance of frame selection, perfect alignment of the optical center of the lens with the pupil center, and advise patients on how to correctly wear their spectacles ${ }^{1,7}$. However, a general observation of the multitude of frame styles and fits being worn by patients today and the reported symptoms associated with induced prism in the literature led the researchers to investigate the prevalence of inappropriately fitting frames and the effect of these on the optical performance of their respective lenses. The aim of this study was to determine the prevalence and the associated prismatic effects of poorly fitting spectacle frames. The specific objectives of the study were to determine the percentage of spectacle wearers whose frames do not fit properly, the prevalence of induced horizontal and vertical prismatic effects in participants not looking through the optical centers of their spectacle lenses, the participants' knowledge on the importance of properly fitted spectacles and lastly the relationship between induced prism and the participants' symptoms.

\section{Methodology}

Ethical clearance to conduct the study was obtained from the University of KwaZulu - Natal (UKZN) Faculty of Health Science Research Committee. A quantitative, descriptive study design was utilized. From a study population of students at the UKZN Westville campus, a study sample of 100 students wearing spectacles for refractive correction were selected. The study sample comprised of a conveniently selected group of students from across the campus. This sample included participants of different race groups and males and females were included. Participants with a recently compensated refractive error and wearing single vision spectacles were included in the study and individuals with a refractive error wearing bifocal or multifocal spectacles and participants with a strabismus were excluded from the study.

A standardization workshop was conducted to 
standardize techniques used and ensure that each of the researchers obtained accurate readings with all techniques. This was followed by a pilot study, conducted on a convenient sample of 10 students, to assess the validity of the questionnaire and refine techniques used. A consent form in English or IsiZulu containing information relating to the purpose, significance and intended procedures of the research study was completed and signed by each participant. Participants completed a questionnaire providing information on their visual and ocular complaints, date of the last visual examination and knowledge on the importance of properly fitting spectacle frames. A unilateral cover test was conducted to rule out any strabismus. An initial observation of the position of the participant's spectacles was made whilst being worn in the habitual position in primary gaze. This subjective observation was noted on the participant's result sheet and photographs of poorly fitting frames were randomly taken.

With the frames in the habitual wearing position, the pupil centers were marked with a blue ink marker and thereafter the distance IPD was measured. The frame PD was measured and compared to the distance IPD to ascertain the face form tilt which was recorded on the result sheets. The optical centers of each of the lenses were then marked with a black colour marker using a vertometer. The distances between these markings were then measured to determine the centration distance. The power of each spectacle lens was then determined using the Iso-Lys vertometer. The oblique cylinders were initially resolved into horizontal and vertical components. Any prismatic effects present were thereafter calculated and recorded using Prentice's Rule.

Data was captured using SPSS Version 15 and Microsoft Excel 2007. The prevalence of poorly fitted frames was derived from the data. A descriptive analysis was done using the ANOVA Kruskal-Wallis and Mann-Whitney Test, with a probability of $p<0.05$ to correlate the participant's symptoms and the induced prism as well as to correlate the refractive errors and the induced prism. To maintain confidentiality the use of a code rather than the participant's name was employed.

\section{Results}

The majority $(63 \%)$ of the participants claimed that they were not informed by their eye-care practitioners and hence were unaware of the importance of properly fitting spectacles (Figure 1).

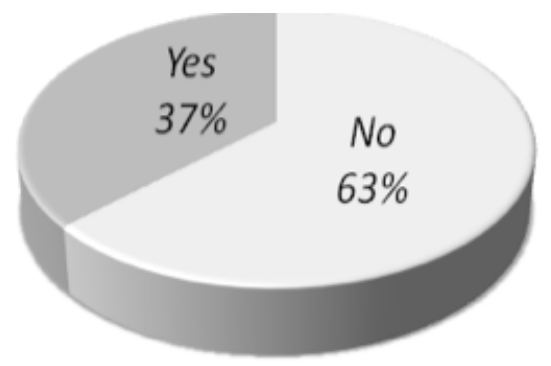

Figure 1: showing the subjects awareness of the importance of properly fitted spectacles.

Forty five percent of the study population was symptomatic with the most common visual complaint being blurry vision $(28 \%)$ and other symptoms experienced included asthenopia (12\%) and headaches $(5 \%)$.

\section{Clinical Findings}

Figure 2 shows that on observation $45 \%$ of subjects appeared to be wearing improperly fitted spectacles, however, a comparison of the marked pupil and optical centers revealed that $100 \%$ of subjects were not looking through the optical centers of their spectacle lenses. Participants with base out prismatic effects experienced the most symptoms (50\%), although there was no correlation between the amount of induced prism and the symptoms experienced. Fifty one percent of the induced prism fell within the horizontal and 3.12\% within vertical ANSI tolerances.

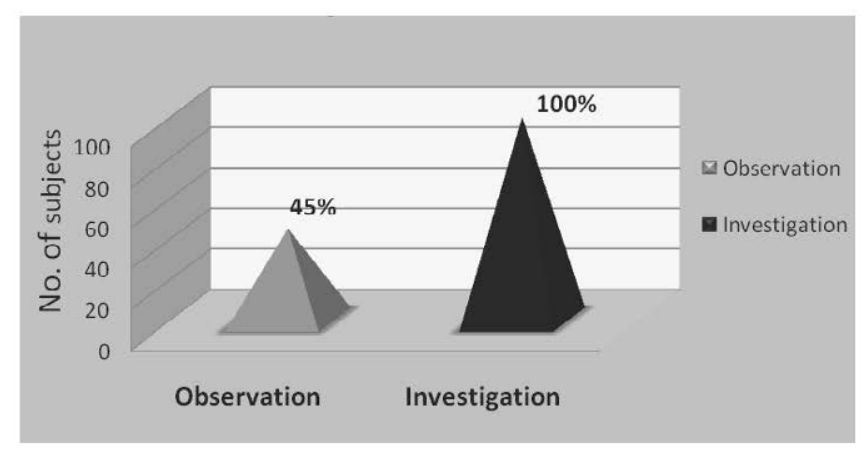

Figure 2: showing the percentages of subjects not looking through the optical centers of their lenses as observed and measured. 


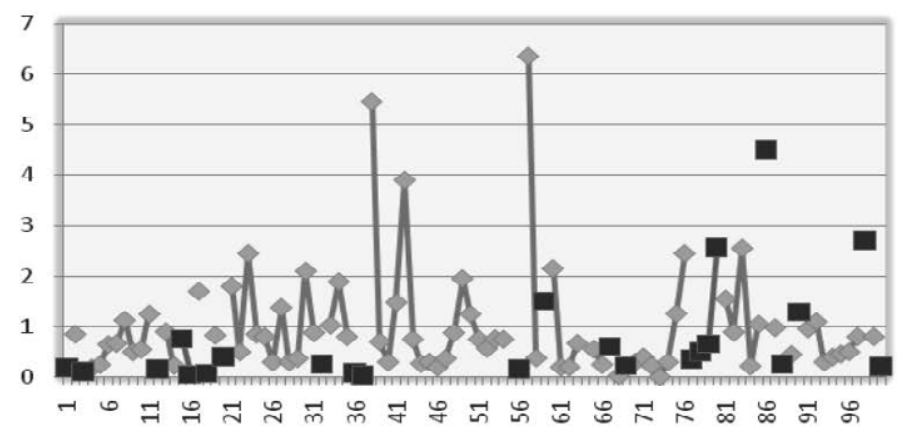

Number of participants

Figure 3: showing the ranges of the induced BI and BO horizontal prismatic effects experienced by participants $(\mathrm{ANSI}=0.75$ $\Delta)$.

Whilst viewing in the primary position $49 \%$ of the participants experienced horizontal induced prisms with the majority of subjects (75\%) experiencing base out prismatic effects in the range $<1 \mathrm{pd}$. The induced prism in these cases could have resulted from myopic patients looking through a point temporal to the distance optical centers or hyperopic patients looking through a point nasal to the distance optical centers. Twenty three percent of subjects experienced base in prismatic effects (Figure 3). Figure 4 shows that $96.8 \%$ of participants experienced vertical prismatic effects with the majority falling in the $<1$ pd range. The vertical induced prism was not further classified in this study.

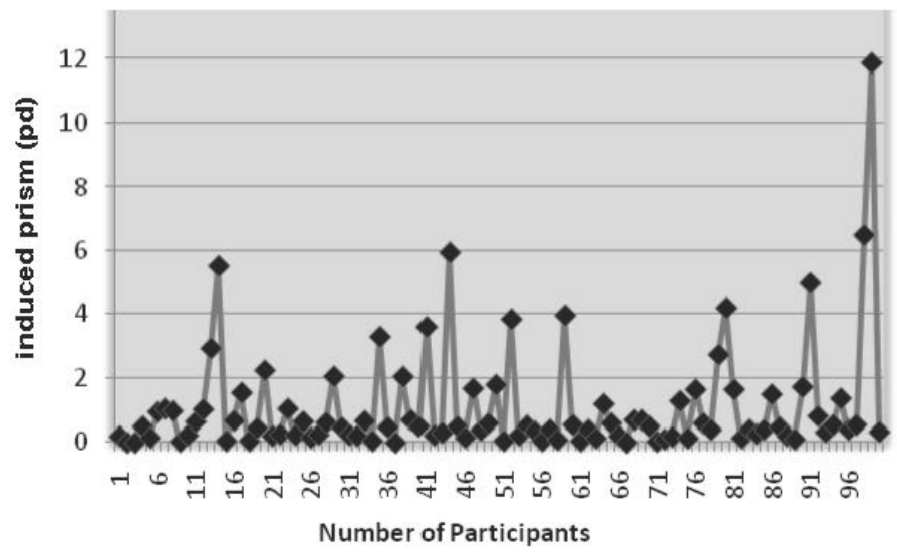

Figure 4: showing the ranges of the induced vertical prismatic effects experienced by participants $($ ANSI $=0.25 \Delta)$.

\section{Discussion}

Despite the majority of the subjects' frames appearing to fit properly on naked eye observation, when measured, a clinically significant number of the sample was found to be wearing their spectacles incorrectly, with all subjects not looking through the optical center of their lenses. This could be avoided by practitioners ensuring proper alignment during the dispensing of the spectacles and providing good patient education which, as shown in this study, is not always done by practitioners. If the laboratory made an error with the spectacles then practitioners should reject them as indicated by Fowler et $a l^{21}$ who state that if a pair of spectacles does not conform to the tolerances then the work should be rejected. One cannot rely solely on patients to ensure that frames fit well to avoid induced prism if the proper alignment of the optical centres are not initially confirmed by the practitioner prior to dispensing the lenses. The poorly fitting frames in this study resulted in all subjects being exposed to a certain amount of induced prism in primary gaze and according to Osubeni ${ }^{9}$ they may experience symptoms such as asthenopia and blurry vision. This is also supported by Topliss ${ }^{15}$ who states that centration is important with modern large lens sizes or the patient will have to overcome excessive prismatic imbalances in order to see the object clearly. In some cases this may cause uncomfortable vision and headaches. This was further confirmed in this study where $45 \%$ of the subjects reported symptoms such as blurry vision, asthenopia and headaches. As shown in Figures 3 and 4, 49\% of the subjects experienced induced horizontal and $96.8 \%$ vertical prismatic effects respectively, both of which were greater than the accepted ANSI tolerance levels. There was however no correlation between the amount of induced prism and the symptoms reported by the participants. Literature $25-27$ indicates that the possible reason for those who were asymptomatic, despite experiencing induced prism that exceeded acceptable tolerances, could be prism adaptation. According to Griffin and Grisham ${ }^{25}$ heterophoric patients having normal binocular vision with no ocular symptoms typically show strong prism adaptation. The symptoms that were reported, largely by participants with induced base out prism, may be as a result of the induced prism compounding other possible near vision anomalies such as convergence insufficiency.

The expected clinical protocol to be performed 
by optometrists during the dispensing of spectacles is to ensure that the patient is looking through the optical center and assuring proper frame alignment. As noted, and of concern in the study, was that the majority of participants' frames were not aligned for proper optical benefit and patients were not adequately educated about the importance of the spectacle frame being well aligned on the face. To ensure that patient care is not compromised practitioners must adhere to the minimum standards of optical dispensing practice.

\section{Conclusion and Recommendations}

This study showed that all the subjects were not looking through the optical centers of their spectacles and most claimed that they were not made aware by the practitioners of the need for proper frame fitting. The pantoscopic tilt, which could have also contributed to the induced prism, was not taken into consideration as the study focused only on the centration of the lenses. Practitioners should however check the pantoscopic tilt as well as centration when dispensing the spectacle frame. Proper optical dispensing practices, accompanied by appropriate patient education is a critical part of the visual consultation and careful attention should be given to this aspect by practitioners. It is thus recommended that patients return periodically to their optometrists to have the frame alignment assessed and the fit modified if necessary. Consideration should be given to include this as part of the minimum standards for optical dispensing practice.

Recommendations for further studies will be to use a larger sample size, include the effects of pantoscopic and face form tilts, determine the effects of the

\section{References}

1. Brooks CW, Borish IM. System for ophthalmic dispensing. New York: Professional Press, 1979 pp 27-65.

2. Tang CY, Tang N, Stewart SC. Facial measurements for frame design. Optom Vis Sci 1998 75(4) 288-92.

3. Obstfeld H. Spectacle frames and their dispensing. Saunders London 1997 pp 136-40.

4. Stimson L. Ophthalmic dispensing 3rd ed. Charles C Thomas, Springfield 1979.

5. Sasieni LS. Principles and practice of optical dispensing and fitting. 3rd ed. Butterworths London 1975 pp 45-185.

6. Dowaliby M. Practical aspects of ophthalmic optics. 4th ed. Butterworth-Heinemann, 2000 pp 229-231.

7. Borish IM. Clinical refraction. 3rd ed. Vol 1 New York:
Professional Press 1970 pp 432-28.

8. Fannin TE, Grosvenor T. Clinical optics 2nd ed. Butterworth-Heinemann Boston 1987 pp 298-300.

9. Osuobeni EP, Al-Fahdi M. Differences between anatomical and physiological interpupillary distance. J Am Optom Assoc 199465 265-71.

10. Jalie M. Ophthalmic lenses and dispensing. 2nd ed. Butterworth-Heinemann London 2003 pp 39-52.

11. Khurana AK. Theory and practice of optics and refraction. 2nd ed. Elsevier, India 2008 pp190- 191.

12. Millodot M. Dictionary of optometry and visual science. 7th ed. Butterworth-Heinemann2009.

13. Wakefield KG. Bennett's ophthalmic prescription work. 4th ed. Butterworth Heinemann Oxford, 2000.

14. Shukla AV. Clinical Optics Primer for Ophthalmic Medical Personnel: A Guide to Laws, Formulae, Calculations and Clinical Applications. SLACK Inc. USA 1950 pp 174-176.

15. Topliss WS. Optical dispensing \& workshop practice. Butterworths London, 1975 pp 42-59.

16. Jalie M. The principles of ophthalmic lenses. 4th ed. The Association of British Dispensing Opticians London 1992.

17. Jiménez JR, Rubiño M, Díaz JA, Hita E, del Barco L. Changes in stereoscopic depth perception caused by decentration of spectacle lenses. Optom Vis Sci 2000 77(8) 421-7.

18. Fogt N, Jones R. The effect of refractive lenses on perceived direction. Vision Res 1996 36(22) 3735-41.

19. Fry GA, Kent PR. The effects of base-in and base-out prisms on stereo-acuity. Am J Optom 194421 492-507.

20. Du Toit R, Ramke J, Brian G. Tolerance to prism induced by readymade spectacles: setting and using standard. $\mathrm{Am} \mathrm{J}$ Optom 2007 84(11) 1053-9.

21. Fowler C, Latham Petre K. Spectacle lenses: Theory and practice. Butterworth-Heinemann Oxford 2001 pp37-51.

22. American Standards Association. ANSI Tolerances. Available from: http://www.drdrbill.com/downloads/optics/ ophth-optics/Standards.pdf (Accessed on the 02/09/2010)

23. Fernández-Ruiz J, Díaz R. Prism adaptation and aftereffect: specifying the properties of a procedural memory system. Learn Mem 1999 6(1) 47-53.

24. Teachers Eye Care. Proper Care of Your Spectacles. Available from: http://www.teacherseyecare.com.au/nbspnbspProducts/ProperCareofyourSpectacles/tabid/62/Default. aspx. (Accessed on the 02/09/2010).

25. Griffin JR, Grisham JD. Binocular anomalies diagnosis and vision therapy, 4th ed. Butterworth Heinemann 2002 p92.

26. Evans BJW. Binocular vision anomalies. Diagnosis and vision therapy. 4th ed. Butterworth Heinemann. 2002 p262.

27. Henson DB, North R. Adaptation to prism induced heterophoria. Am J Optom Physiol Opt 198057 129-137. 